\title{
A Numerical Simulation of the Deformation and Fracture of a Material with a Porous Polysilazane Coating
}

\author{
Ruslan R. Balokhonov ${ }^{1,2, a)}$, Aleksandr V. Zinoviev, b), Varvara A. Romanova ${ }^{1,2, c)}$, \\ and Sergei A. Martynov, d) \\ ${ }^{1}$ Institute of Strength Physics and Materials Science SB RAS, Tomsk, 634055, Russia \\ ${ }^{2}$ National Research Tomsk Polytechnic University, Tomsk, 634050, Russia

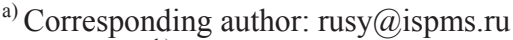 \\ b) zav@ispms.ru \\ c) varvara@ispms.ru \\ d) martynov@ispms.tsc.r
}

\begin{abstract}
A numerical analysis of the deformation and fracture mechanisms involved in a material with a porous ceramic coating under tension and compression is presented. The dynamic boundary-value problem in the plane strain formulation is solved numerically by the finite difference method. To take an explicit account of the substrate-coating interface and porous coating microstructure in the calculations, a curvilinear mesh generation algorithm based on the solution according to elasticity theory has been developed. A two-dimensional curvilinear mesh generated in this work was used to simulate the uniaxial loading of a material with a porous coating. The fundamental difference between the fracture mechanisms operating in the coated material in the cases of tension and compression was found to be related with the formation of local regions experiencing bulk tension in both cases.
\end{abstract}

Keywords: numerical simulation, porous ceramic coatings, plasticity, fracture, curvilinear mesh

\section{INTRODUCTION}

A description and prediction of the mechanical behavior of coated materials are a topical problem to be solved in a wide range of scientific disciplines and industrial applications. In recent years, light-weight high-strength metalceramic composites have been widely used instead of pure metallic compounds in the most important high-precision industries, such as microelectronics, aviation and space exploration, electric power engineering, etc.

One of the important groups of pre-ceramic polymers is polysilazanes. Due to their optimum reaction with moist and polar surfaces, polysilazanes are employed to produce fibers, coatings and fiber-reinforced ceramic-matrix composites. The use of ceramic coatings based on polysilazanes insures optimum adhesion. For certain applications, however, such coatings are too thin and porous [1]. There is an increasing number of experimental and numerical studies aimed at investigating the shape, microstructure and spatial distribution of pores and at analyzing methods for estimating the porosity and producing porous coatings (see, e.g., [2]).

This paper discusses the mechanisms of deformation and fracture experienced by a material with a porous polysilazane-based ceramic coating subjected to tension and compression. The simulated microstructure of the coated material corresponds to that produced experimentally (Fig. 1) and is accounted for explicitly in the calculations. To simulate the mechanical responses of the substrate and coating materials, use was made of an elasto-plastic model accounting for strain hardening and of a modified fracture criterion of the Huber type, respectively [3].

The work consists of two stages. In stage 1, a 2D regular curvilinear mesh generation algorithm based on the method proposed in [4] is designed, and a pertinent finite-difference solution is compared with that obtained with the use of a uniform rectilinear mesh (see, e.g., $[3,5])$. The curvilinear mesh plays an important role in describing real pore shapes and those of the curvilinear coating-substrate interface. Stage 2 involves simulations of the deformation and fracture of the coated material under compression or tension at the mesoscale level (Fig. 1).

International Conference on Physical Mesomechanics of Multilevel Systems 2014

AIP Conf. Proc. 1623, 51-54 (2014); doi: 10.1063/1.4898880

(C) 2014 AIP Publishing LLC 978-0-7354-1260-6/\$30.00 


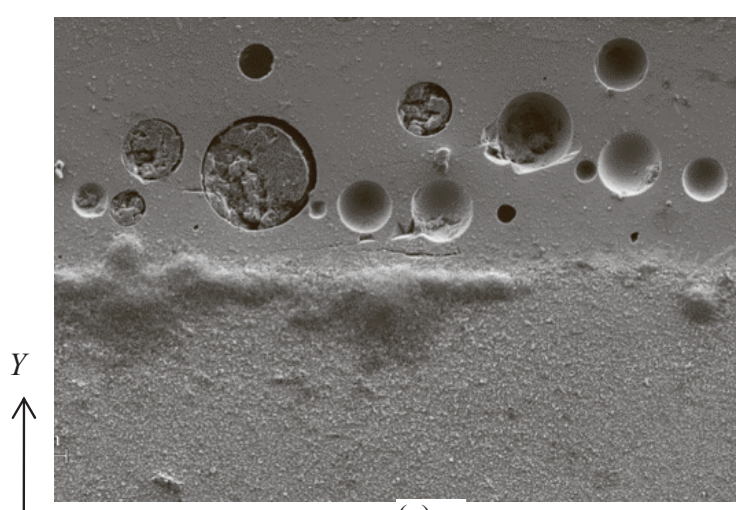

(a)

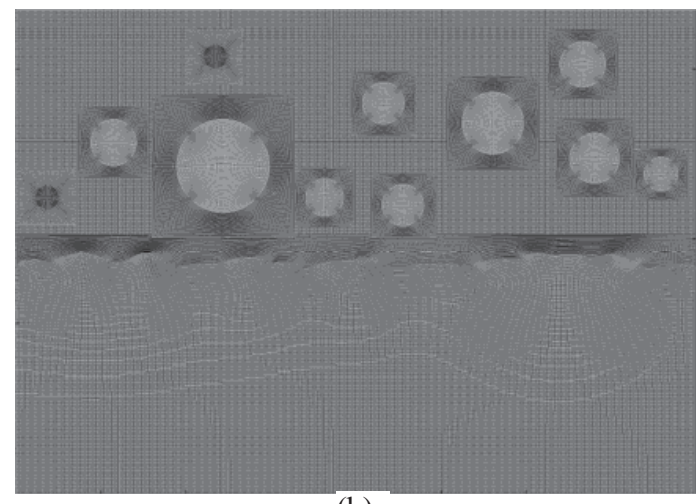

(b)

FIGURE 1. Scanning electron microscope image of a material with a porous ceramic coating (a) [1] and a regular curvilinear mesh $(b)$

\section{AN ALGORITHM FOR 2D CURVILINEAR MESH GENERATION}

In our earlier work, we used regular rectilinear meshes describing interfaces in a stepwise manner, i.e., a curvilinear interface was represented as a set of straight lines joined together at right angles (Fig. 2(a)). To generate 2D regular curvilinear meshes describing interfaces in a smoother and more accurate way, we solved the quasi-static boundary-value problem in the plane stress formulation [5]. The elasticity problem expressed in terms of displacements as

$$
\begin{aligned}
& \frac{\partial^{2} u_{x}}{\partial x^{2}}+\frac{1-\vartheta}{2} \frac{\partial^{2} u_{x}}{\partial y^{2}}+\frac{1+\vartheta}{2} \frac{\partial^{2} u_{y}}{\partial x \partial y}=0 \\
& \frac{\partial^{2} u_{y}}{\partial y^{2}}+\frac{1-\vartheta}{2} \frac{\partial^{2} u_{y}}{\partial x^{2}}+\frac{1+\vartheta}{2} \frac{\partial^{2} u_{x}}{\partial x \partial y}=0
\end{aligned}
$$

was used to simulate the deformation of the initially regular rectangular mesh [4]. It should be pointed out that the simulation has no physical sense and is used to generate a finite-difference mesh. Here $\vartheta$ is not Poison's ratio. This is a computational parameter varying between 0 and 1. Equations (1) were solved numerically by the finitedifference Seidel method. Figure 2(b) shows curvilinear mesh generation results for a rectangular region with a single perfectly round pore at the center. Boundary conditions in terms of displacements were assigned to the dashed boundary of the square region to obtain round interfaces. The distribution of cell volumes is seen to be fairly uniform. The pore size can be varied by stretching or compressing the dashed square region. The algorithm was applied to two-dimensional curvilinear mesh generation for an experimental microstructure of a coated material containing multiple pores in the coating and a curvilinear coating-substrate interface (Fig. 1(b)).

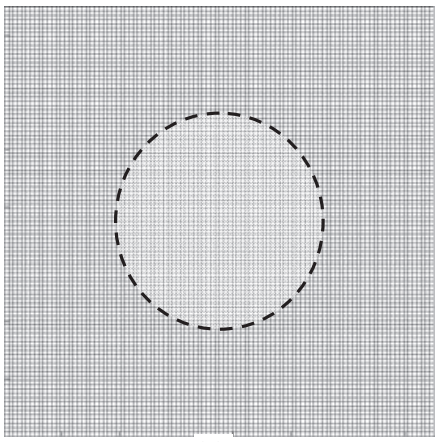

(a)

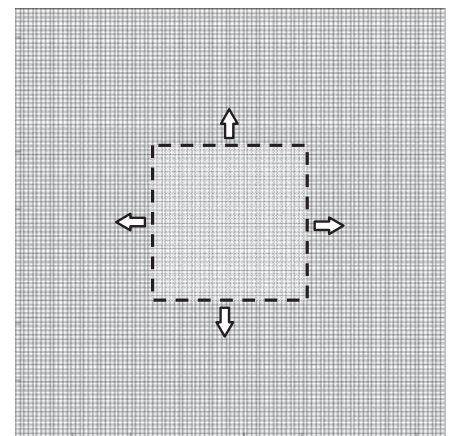

(b)

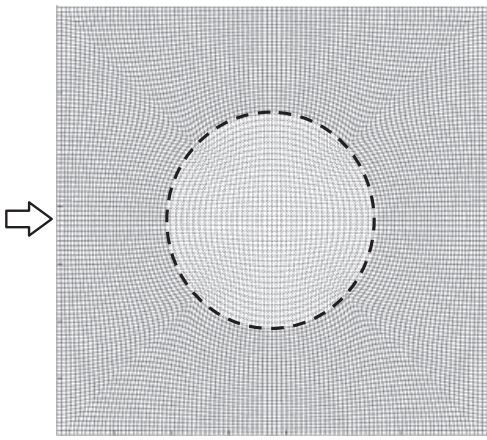

FIGURE 2. Rectilinear (a) and curvilinear meshes (b) for a region with a single pore 


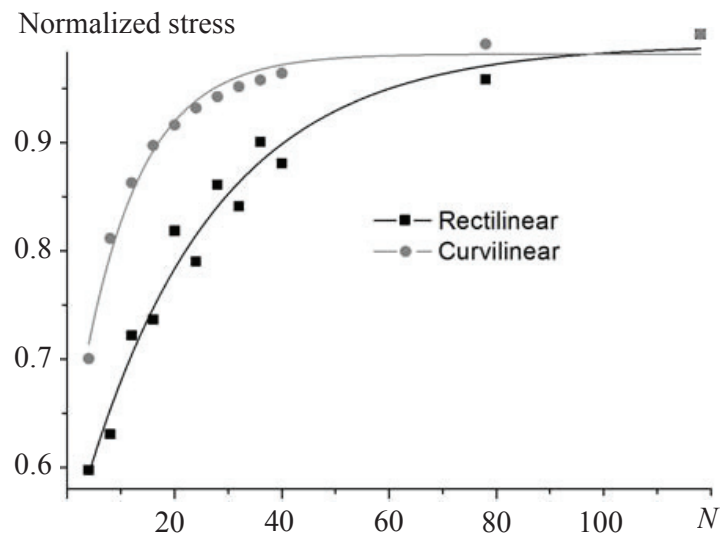

FIGURE 3. Maximum equivalent stress vs. the number of nodes per pore diameter $N$

\section{A NUMERICAL SIMULATION OF THE DEFORMATION OF A COATED MATERIAL}

\section{A Mathematical Formulation of a Mechanical Problem}

The mesoscopic deformation of a material with a porous coating was simulated in the plane strain formulation. A complete system of equations includes equations of motion and continuity, expressions for the strain rate tensor components and constitutive relations $[3,5]$. The dynamic boundary-value problem was solved numerically, using the finite-difference method [6]. The substrate material exhibited an elastic-plastic response. The plastic flow rule used is associated with the yield function taking into account isotropic strain hardening. The coating is elasic-brittle ceramics. To describe the fracture of the coating material, use was made of a maximum distortion energy criterion accounting for crack initiation and growth in local regions experiencing bulk tension. According to this criterion, fracture occurs if the equivalent stress reaches a critical value and the cubic strain takes on a positive value in the local region.

\section{Convergence of the Numerical Solution for a Rectangular Region with a Single Pore}

Two sets of numerical experiments have been performed for rectilinear and curvilinear meshes (see Fig. 2) approximating a rectangular region of the coating material with a perfectly round pore under tension. In the calculations, the number of cells per pore diameter was varied, with the pore size being the same. The cell material inside the pore possessed very low elastic properties. The calculation results are shown in Fig. 3. Convergence of the numerical solution was found for both cases. The use of the curvilinear mesh is seen to accelerate the approach of a maximum stress concentration to a saturation physical value dictated by the geometry of the pore material and independent of the mesh size (Fig. 3).

\section{The Deformation and Fracture of a Steel Substrate with a Porous Ceramic Coating}

The finite-difference numerical simulations of the mechanical behavior of the structure of the coated material (Fig. 1(a)) approximated by the curvilinear mesh (Fig. 1(b)) have been performed for uniaxial tension and compression. In the case of tension or compression, the tensile and compressive stresses are found to occur along pore surfaces (Fig. 4(a)), whereas cracks originate in the local regions experiencing tensile loading in both cases. In tension and compression, tensile regions are located at different points. That is why the fracture patterns depend heavily on the type of the external loading, with all other conditions being equal.

Under tension, cracks in polysilazine originate near the top or bottom pore surface and propagate perpendicular to the direction of load application both toward the coating surface and toward the coating-substrate interface (Fig. 4(b)). On further loading the plasticity of the substrate material prevents the crack from propagating deep into the material, and it sets in motion along the interface (Fig. 4(c)). Part of the coating separates from the substrate, which causes the coating to lose its functionality and protective properties (Fig. 4(c)). 

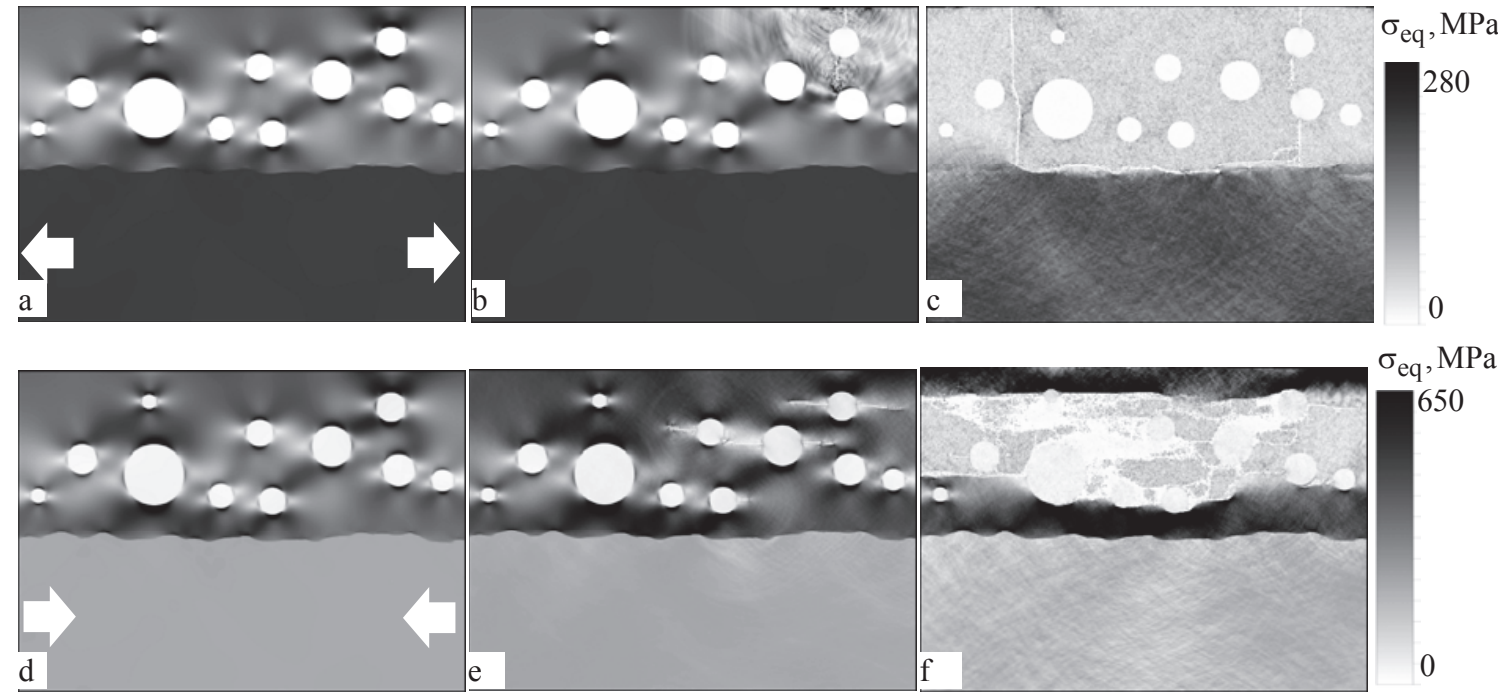

FIGURE 4. Equivalent stress patterns under tension ( $a, b$ and $c)$ and compression $(d, e$ and $f)$ of the coated material

Under compression, the fracture of the coated material occurs at a higher degree of the total strain as compared to tension (cf. Figs. 4(a and d)), which agrees in qualitative terms with well-known experimental evidence for brittle materials and composites. Cracks originate near the left- or right-hand pore surfaces and propagate from pore to pore along the direction of load application, and layer-by-layer delamination of the coating material takes place. Late in the process, a thin pore-free polysilazane layer deposits on the substrate. As a result, the functionality and protective properties of the coating are retained in part.

\section{ACKNOWLEDGEMENTS}

The work was supported by grants from a program to increase the competence of TPU and Russian Science Foundation (grant No. 14-19-00766).

\section{REFERENCES}

1. A. Schütz, M. Günthner, G. Motz, O. Greißl, and U. Glatzel, Surf. Coat. Technol. 33, 319 (2012).

2. D. H. Kohn, Compr. Biomater. 6, 65 (2011).

3. R. R. Balokhonov, V. A. Romanova, S. Schmauder, S. A. Martynov, and Zh. G. Kovalevskaya, Composites: Part B 66, 276 (2014).

4. A. A. Ignatiev, Mat. Modelir. 12, 101 (2000).

5. R. R. Balokhonov, V. A. Romanova, S. Schmauder, and P. V. Makarov, Comp. Mater. Sci. 28, 505 (2003).

6. M. Wilkins, Computer Simulation of Dynamic Phenomena (Springer-Verlag, Berlin, 1999). 